Molecules 2007, 12, 2559-2566

molecules

ISSN 1420-3049

(C) 2007 by MDPI

www.mdpi.org/molecules

Full Paper

\title{
The Drug Release Profile from Calcium-induced Alginate Gel Beads Coated with an Alginate Hydrolysate
}

\author{
Yoshifumi Murata ${ }^{1 . *}$, Daisaku Jinno ${ }^{1}$, Dongchun Liu ${ }^{2}$, Takshi Isobe ${ }^{1}$, Kyouko Kofuji ${ }^{1}$ and \\ Susumu Kawashima ${ }^{1}$ \\ ${ }^{1}$ Faculty of Pharmaceutical Science, Hokuriku University, Ho-3, Kanagawa-machi, Kanazawa \\ 920-1181, Japan \\ ${ }^{2}$ Department of Pharmacognosy, Shenyang Pharmaceutical University, Wen Hua Road 103, mailbox \\ 48, Shenhe, Shenyang, 110016, PRC
}

* Author to whom correspondence should be addressed; E-mail: y-murata@hokuriku-u.ac.jp; Phone: +81-076-229-6184; Fax: +81-076-229-2781

Received: 17 November 2007; in revised form: 27 November / Accepted: 27 November 2007 / Published: 29 November 2007

\begin{abstract}
Calcium-induced alginate gel bead (Alg-Ca) coated with an alginate hydrolysate (Alg), e.g. the guluronic acid block (GB) was prepared and the model drug, hydrocortisone release profiles were investigated under simulated gastrointestinal conditions. Their molecular weights were one sixth or one tenth that of Alg and the diffraction patterns of the hydrolysates resembled that of Alg. The drug release rate from Alg-Ca coated with GB apparently lowered than that of Alg-Ca (coating-free) in the gastric juice (pH1.2). And the coating did not resist the disintegration of Alg-Ca in the intestinal juice ( $\mathrm{pH} 6.8)$ and the gel erosion accelerated the drug release. On the other hand, for the coated Alg-Ca containing chitosan, the drug release showed zero-order kinetics without rapid erosion of Alg-Ca. The drug release rate from Alg-Ca was able to be controlled by the coating and modifying the composition of the gel matrix.
\end{abstract}


Keywords: Alginate hydrolysate, Alginate gel bead, Gel bead coating, Drug release profile.

\section{Introduction}

Alginic acid (Alg), a natural anionic polysaccharide, has been used as a medicine for stomach ulcers as well as a food additive because of the protective effect for the gastric mucosa on per oral administration. The solution of sodium alginate immediately forms a cured gel matrix in the presence of a divalent or a trivalent cation and this characteristic has been utilized practically as a bioreactor $[1,2]$. In addtion calcium-induced alginate gel beads (Alg-Ca) are noted as a vehicle for drug delivery [3-5]. Alg consists of $\alpha-L$-guluronic acid (G) and $\beta$-D-mannuronic acid (M), and it is known that the composition of Alg, such as $\mathrm{M} / \mathrm{G}$ ratio changes the characteristics of the Alg-Ca prepared with the Alg [6]. A sequence of G-G, G-blocks (GB) in the Alg molecule is closely related with the gel matrix formed between Alg and calcium ions. The drug release profile from Alg-Ca is affected by both the structure of the gel matrix and the properties of drug itself, such as its solubility.

GB is obtained by the hydrolysis of Alg and is itself also a polyanionic compound [7]. We have reported that the rheological properties of the Alg-Ca and the drug release profile from the beads were affected by the addition of GB to the gel matrix [8]. When dried Alg-Ca is administered orally, the gastrointestinal fluid permeates into the gel matrix, and then the drug incorporated in Alg-Ca is gradually released from the vehicle through the watercourse formed by the penetration. Therefore, the characteristic of the drug release from Alg-Ca may be controlled by the modification of the gel matrix if GB incorporated in Alg-Ca behaves as a component of the gel matrix [9,10]. Especially, the gel-matrix structure of Alg-Ca surface is very important for the release profile of drug contained in the vehicle. In the present study, we attempted to prepare Alg-Ca coated with Alg hydrolysate by the simple method in the aqueous solution, and investigated the drug release profile from the preparation under simulated gastrointestinal conditions.

\section{Results and Discussion}

The Alg hydrolysates were obtained by partial degradation with dilute $\mathrm{HCl}$. The results of the gel permeation chromatography of Alg and the hydrolysates are shown in Table 1. The molecular weight of GB or a M-M, M-block sequence (M) was one sixth or one tenth that of Alg, respectively. As shown in Figure 1, Alg exhibited a characteristic amorphous polymer X-ray diffraction pattern. The diffraction pattern of GB or MB resembled that of Alg, i.e., "a halo pattern” [11]. These results show that $\mathrm{GB}$ or $\mathrm{MB}$ is a low molecular weight Alg, thus a $5 \%$ solution of the hydrolysate did not form cured hydrogel beads in $\mathrm{CaCl}_{2}$. 
Table 1: Molecular weight of alginate and the hydrlysates.

\begin{tabular}{|l|l|}
\hline Sample & M.W. (Dispersion) \\
\hline Alg & $188,000(4.1)$ \\
GB & $22,700(2.9)$ \\
MB & $13,400(3.3)$ \\
\hline
\end{tabular}

Since the Alg solution is viscous; e.g., $52 \pm 2 \mathrm{cp}$ at $1 \%$, some hydrogel beads gradually stuck together after Alg-Ca was transferred to $1 \%$ of Alg solution for coating the surface. On the other hand, the viscosity of $5 \% \mathrm{~GB}$ or MB solution was below $10 \mathrm{cp}$, and coagulation did not occur among the beads during the coating period. The treatment of Alg-Ca with 1-5\% of GB solution changed the surface structure of the gel matrix. When Alg-Ca (hydrogel) is transferred to the GB solution, calcium ions existed in Alg-Ca diffuse to the surface, and then bind with GB and form the film-like gel on the surface. As shown in Figure 2, the surface of Alg-Ca treated with GB solution was coated with a transparent gel layer and it was smoother than that of Alg-Ca.

Figure 1. X-ray diffractograms of (a) Alg, (b) GB and (c) MB.

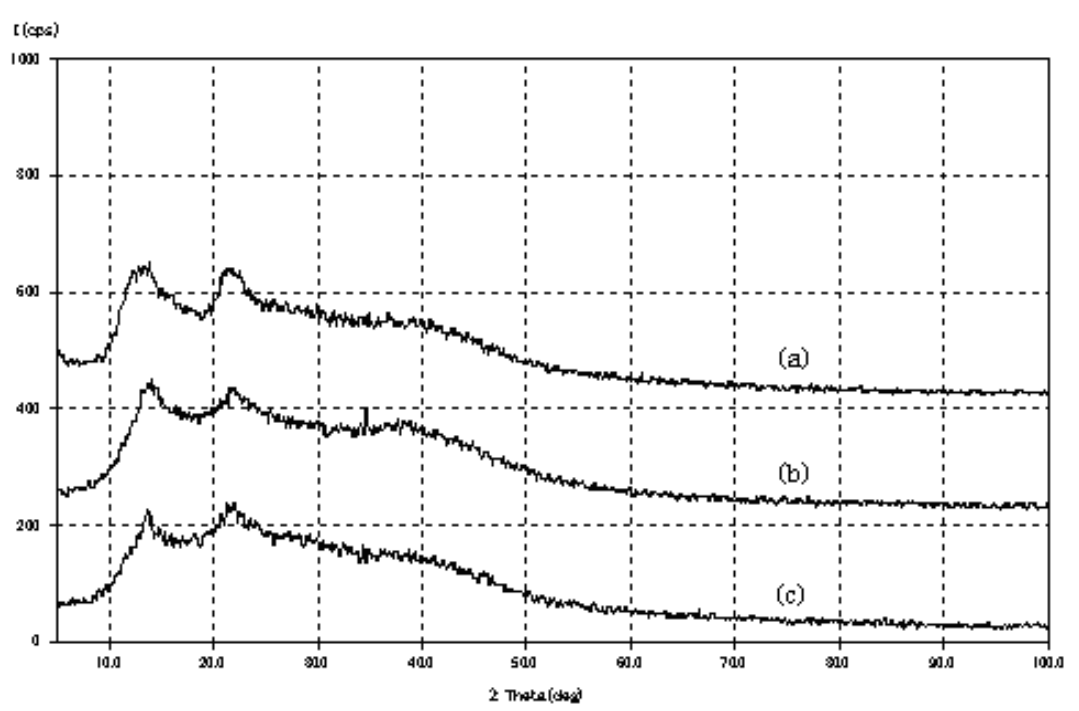

Figure 2. Photograph of dried Alg-Ca.

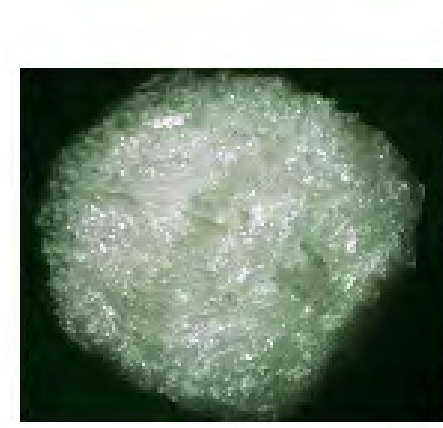

(A)

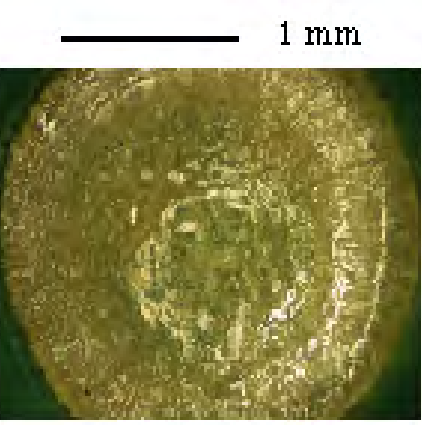

(B)

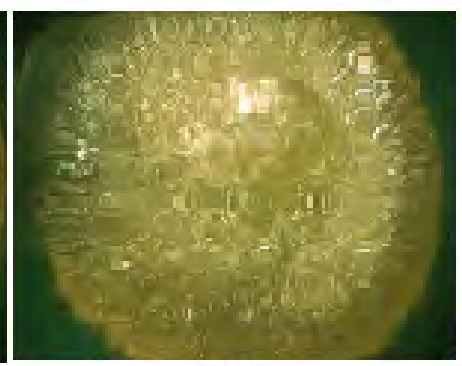

(C)

(A) Alg-Ca, (B) Alg-Ca coated with 1\% GB, (C) Alg-Ca coated with 1\% MB. 
The amount of the model drug, hydrocortisone (HC) incorporated in Alg-Ca decreased slightly owing to the coating process, as shown in Figure 3. This may be attributed to leakge of the HC held in the alginate gel matrix to the GB solution. The loss of drug incorporated in Alg-Ca was suppressed by addition of GB to Alg that is raw material for the formation of Alg-Ca. For example, the incorporation of $\mathrm{HC}$ in Alg-Ca prepared with Alg containing 5\% GB was $78.3 \pm 0.3 \%(n=3)$. However, Alg-Ca (containing 5\% GB) coated with 5\% GB slightly decreased the HC incorporation with respect to 1\% of the same coating as shown in Figure 3 (c and d). This phenomenon may be attributed to a scramble for to get $\mathrm{Ca}^{2+}$ between GB contained in Alg-Ca and one used for coating of the beads.

Figure 3. Incorporation of HC in Alg-Ca.

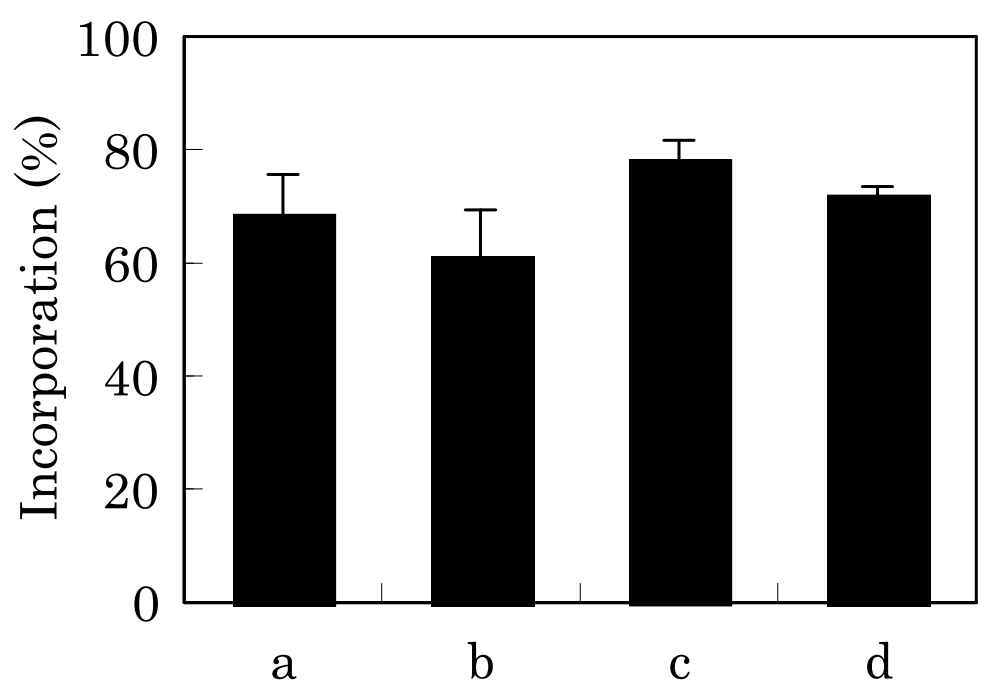

The data are presented as mean value $\pm \mathrm{SD}(\mathrm{n}=3)$.

a; Alg-Ca (coating-free)

b; Alg-Ca (coated with 1\% GB)

c; Alg-Ca containing 5\% GB (coated with 1\% GB)

d; Alg-Ca containing 5\% GB (coated with 5\% GB)

The gel matrix formed between GB and calcium ion on the surface of Alg-Ca affected the drug release profile from the vehicle. In the 1st fluid, the drug release rate from Alg-Ca treated with GB was apparently lower than that of Alg-Ca, as shown in Figure 4. Alg-Ca treated with 1\% GB released $7.9 \pm 0.9 \%$ of the incorporated HC after 120 min [Figure 4(a)]. This result suggests that $\mathrm{Ca}^{2+}-\mathrm{GB}$ chelate acts as the layer, which controls the release rate of $\mathrm{HC}$ from Alg-Ca in the 1st fluid as well as $\mathrm{Ca}^{2+}$ induced Alg gel matrix. And the value was $8.5 \pm 0.7 \%$ in the case of Alg-Ca prepared with Alg containing 5\% GB, then treated with 1\% GB. As shown in Figure 4(b), when Alg-Ca made from Alg containing $5 \% \mathrm{~GB}$ was coated with $5 \% \mathrm{~GB}$, it released $6.3 \pm 0.3 \%$ of $\mathrm{HC}$ after $120 \mathrm{~min}$, and in the case of $5 \% \mathrm{MB}$ coating, this value was $11.2 \pm 0.2 \%$. Irrespective whether Alg-Ca was coated with GB or MB, the coating layer did not resist the disintegration of Alg-Ca in the 2nd fluid ( $\mathrm{pH}$ 6.8) and the gel erosion accelerated the drug release. 
Figure 4. HC-Release curves from Alg-Ca prepared with $0.1 \mathrm{M} \mathrm{CaCl}_{2}$.
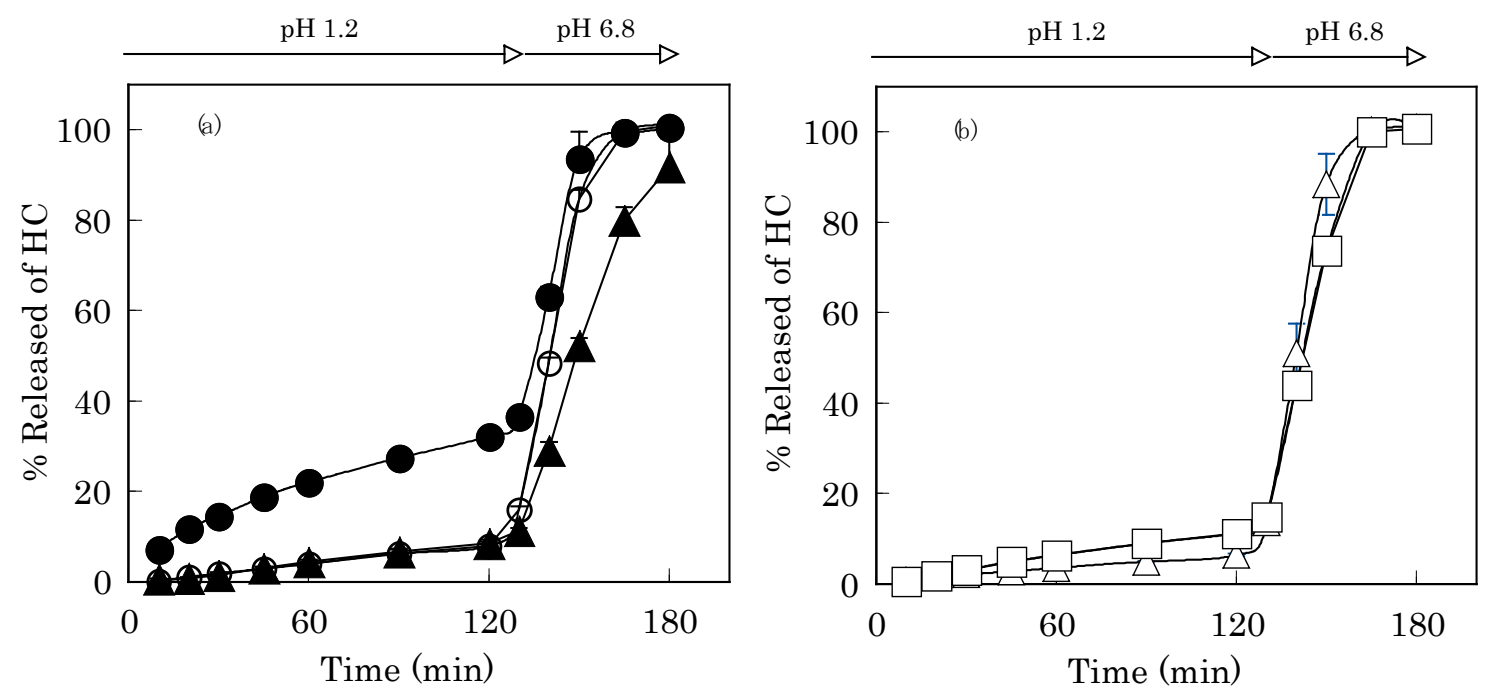

(a) Closed circle; Alg-Ca (coating-free)

(a) Open circle; Alg-Ca (coated with 1\% GB)

(a) Closed triangle; Alg-Ca containing 5\% GB (coated with 1\% GB)

(b) Open triangle; Alg-Ca containing 5\% GB (coated with 5\% GB)

(b) Open square; Alg-Ca containing 5\% GB (coated with 5\% MB)

Addition of the cationic polysaccharide chitosan (CS) to Alg-Ca did not affect the incorporation of HC into the gel matrix (68.6 $\pm 4.2 \%, n=6)$, however, the drug release profile in the 2nd fluid apparently changed. As shown in Figure 5, sustained HC release without rapid erosion of Alg-Ca was noted and this phenomenon may be due to complex formation between Alg (or GB) and CS in Alg-Ca.

Figure 5. HC-Release curves from Alg-Ca containing CS and GB.

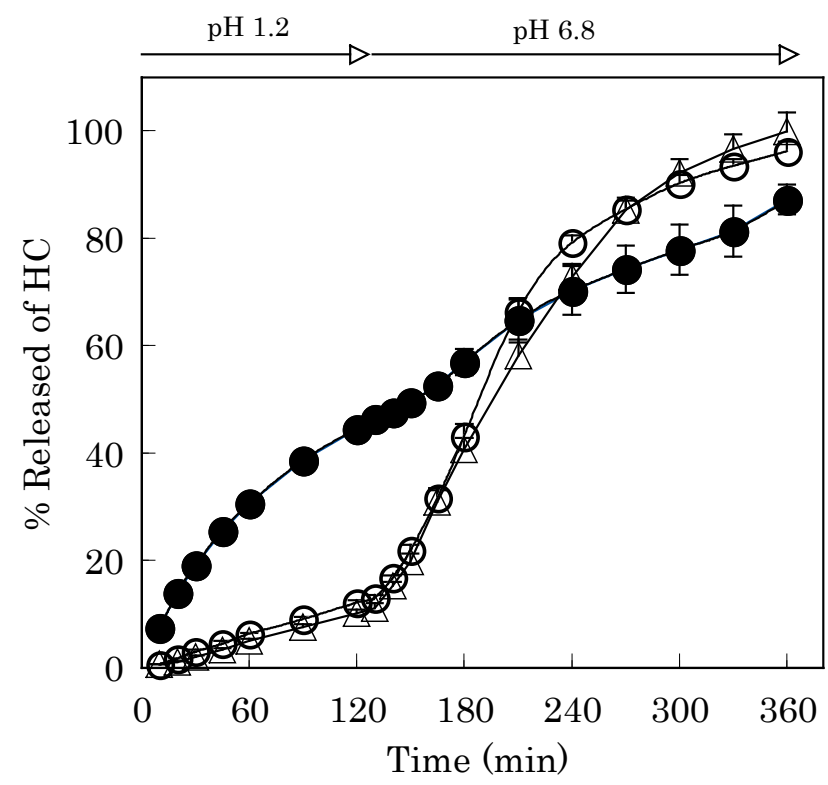

Closed circle; 1\% CS (coating-free). Open circle; 1\% CS and 1\% GB (coated with 1\% GB) .

Open triangle; $1 \%$ CS and 5\% GB (coated with 5\% GB) 
The release of HC from Alg-Ca containing CS showed zero-order kinetics in the 2nd fluid. The apparent release rate was determined as $0.64 \% / \mathrm{min}\left(r^{2}=0.989\right)$, which was calculated by subjecting all the HC dissolution data collected after incubation for 120-240 min from Alg-Ca coated with 1\%GB to least-squares linear fitting. Similar release rate was obtained with Alg-Ca (containing 1\% CS and 5\% GB) coated with $5 \% \mathrm{~GB}\left(0.58 \% / \mathrm{min}, r^{2}=0.990\right)$. Their release rates were faster than that of Alg-Ca (coating-free). This result shows that the complex formed from CS and GB is more fragile in the 2nd fluid than that formed from CS and Alg because GB is a short chain Alg.

\section{Conclusions}

In this study, we prepared Alg-Ca coated with the hydrolysate, after which we investigated the drug release profile. We observed that drug release rate from Alg-Ca could be controlled by the coating and modifying the composition of the matrix. Alg-Ca may be excellent vehicle for oral drug administration due to not only to its safety but also its properties to enable control of drug release in the gastrointestinal tract.

\section{Experimental}

\section{General}

Alg (sodium salt, degree of polymerization; 450) was obtained from Nacalai Tesque (Kyoto), and CS (fine powder, degree of deacetylation; 75-85\%) was obtained from Kimitsu Chem. Industries (Tokyo). HC was purchased from Wako Pure Chemical Ind. (Osaka). All other reagents used were of analytical grade.

\section{Preparation of the hydrolysates}

GB and a sequence of $\mathrm{MB}$ were separated using the method of Haug et al. [7]. The solutions containing the hydrolysates were then neutralized with $\mathrm{NaOH}$, followed by precipitation with ethanol and centrifugation (3000 rpm, $5 \mathrm{~min}$ ). The pellet was washed 3 times with ethanol, and dried in vacuo over $\mathrm{P}_{2} \mathrm{O}_{5}$. The molecular weight of the hydrolysate was determined by gel permeation chromatography [12]. Powder X-ray diffractometry was carried out using an automatic diffractometer $\left(\mathrm{MXP}^{3}\right.$, MAC Science Co., Yokohama) with a voltage of $40 \mathrm{kV}$ and a current of $20 \mathrm{~mA}$. The scanning rate was $2^{\circ} / \mathrm{min}$ over a $2 \theta$ range of $5-100^{\circ}$. The X-ray diffraction results were interpreted using a computer program (XPRESS, Bruker AXS K.K., Yokohama).

\section{Rheological properties}

The viscosity of the solution containing Alg or Alg hydrolysate was measured in triplicate at $37^{\circ} \mathrm{C}$ with a VM-1G-M viscometer (CBC Materials Co., Ltd., Tokyo). 


\section{Preparation of modified alginate gel beads}

Alg-Ca modified by GB and/or CS was prepared as follows: Alg was dissolved in distilled and demineralized water with agitation and GB and/or CS were added to the solution. The model drug (50 $\mathrm{mg}$ ) was then added to the solution (10 g) and mixed until homogeneity. This solution ( $2 \mathrm{~g})$ was then combined with $0.1 \mathrm{M}$ calcium chloride solution $(10 \mathrm{~mL})$ and left standing for $2 \mathrm{~h}$ at room temperature, after which spherical hydrogel beads were obtained. The beads were transferred to $1-5 \%$ hydrolysate solution $(10 \mathrm{~mL})$ and stirred mildly with magnetic stirrer for $1 \mathrm{~h}$ and then they were washed twice with distilled water $(50 \mathrm{~mL})$ and dried at $30^{\circ} \mathrm{C}$ for $8 \mathrm{~h}$ on a culture dish, and they were further dried in a desiccator in the presence of $\mathrm{P}_{2} \mathrm{O}_{5}$. The morphology of Alg-Ca was observed with digital microscope (VHX-200, Keyence Co., Osaka).

\section{Drug release test}

Release of HC incorporated in Alg-Ca was determined in JP XIV 1st medium (pH 1.2, $500 \mathrm{~mL}$ ) for $2 \mathrm{~h}$ and subsequently in 2nd medium ( $\mathrm{pH}$ 6.8) for $4 \mathrm{~h}$ using JP XIV dissolution test apparatus (Toyama Sangyo, Osaka, paddle method, $\left.150 \mathrm{rpm}, 37 \pm 0.5^{\circ} \mathrm{C}\right)$. Release testing was carried out according to previously reported methods [8]. All dissolution tests were performed in triplicate. The solubility of $\mathrm{HC}$ at $37^{\circ} \mathrm{C}$ was $0.38 \mu \mathrm{g} / \mathrm{mL}$ in the $1 \mathrm{st}$ medium or $0.35 \mu \mathrm{g} / \mathrm{mL}$ in the $2 \mathrm{nd}$ medium.

\section{Acknowledgments}

This work was supported in part by a grant (2007) from Hokuriku University.

\section{References}

1. Le-Tien, C.; Millette, M.; Lacroix, M.; Mateescu, M.A. Modified alginate matrices for the immobilization of bioactive agents. Biotechnol. Appl. Biochem. 2004, 39, 189-198.

2. De Alteriis, E.; Silvestro, G.; Poletto, M.; Romano, V.; Capitanio, D.; Compagno, C.; Parascandola, P. Kluyveromyces lactis cells entrapped in Ca-alginate beads for the continuous production of a heterologous glucoamylase. J. Biotechnol. 2004, 109, 83-92.

3. Koelwel, B.F.; Koelwel, C.; Gopferich, A.; Gabler, B.; Wiegrebe, E.; Lohmann, C.P. Tolerance of a new calcium alginate depot for controlled medication of the eye. Ophthalmologe 2004, 101, 496-499.

4. Chai, Y.; Mei, L.H.; Wu, G.L.; Lin, D.Q.; Yao, S.J. Gelation conditions and transport properties of hollow calcium alginate capsules. 2004, Biotech. Bioengineer. 2004, 87, 228-233.

5. Ferreira, A.P.; Almeida, A.J. Cross-linked alginate-gelatine beads: a new matrix for controlled release of pindolol. J. Control. Rel. 2004, 97, 431-439.

6. Kuo, C.K.; Ma, P.X. Ionically crosslinked alginate hydrogels as scaffolds for tissue engineering: part 1. Structure, gelation rate and mechanical properties. Biomaterials 2001, 22, 511-521. 
7. Haug, A.; Larsen, B.; Smidsrod, O. Uronic acid sequence in alginate from different sources. Carbohyd. Res. 1974, 32, 217-225.

8. Murata, Y.; Jinno, D.; Kofuji, K.; Kawashima, S. Properties of calcium-induced gel beads prepared with alginate and hydrolysates. Chem. Pharm. Bull. 2004, 52, 605-607.

9. Shu, X.Z.; Zhu, K.J. The release behavior of brilliant blue from calciu-alginate gel beads coated by chitosan: the preparation method effect. Eur. J. Pharm. Biopharm. 2002, 53, 193-201.

10. Rastello, De Biosseson M.; Leonard, M.; Hubert, P.; Marchal, P.; Stequert, A.; Castel, C.; Favre, E.; Dellacherie, E. Physical alginate hydrogels based on hydrophobic or dual hydrophobic/ionic interactions: bead formation, structure, and stability. J. Colloid. Interface Sci. 2004, 273, 131-139.

11. Puttipipatkhachorn, S.; Nunthanid, J.; Yamamoto, K.; Peck, G.E. Drug physical state and drug-polymer interaction on drug release from chitosan matrix film. J. Control. Rel. 2001, 75, 143-153.

12. Murata, Y.; Miyashita, M.; Kofuji, K.; Miyamoto, E.; Kawashima, S. Drug release properties of a gel bead prepared with pectin and hydrolysate. J. Control. Rel. 2004, 95, 61-66.

Sample Avalibility: Contact the authors.

(C) 2007 by MDPI (http://www.mdpi.org). Reproduction is permitted for noncommercial purposes. 